\title{
Effective $\mathrm{t}-\mathrm{J}$ model of pairing: singlet versus triplet
}

\author{
J.Spałek* \\ Marian Smoluchowski Institute of Physics, Jagiellonian University, \\ Reymonta 4, 30-059 Kraków, Poland
}

Received May 30, 2008, in final form June 18, 2008

\begin{abstract}
The $\mathrm{t}-\mathrm{J}$ model is regarded as a canonical model of spin-singlet pairing induced by the kinetic exchange interaction also responsible for an antiferromagnetic ordering in the strongly correlated narrow-band systems. In the orbitally degenerate systems both ferromagnetic and antiferromagnetic kinetic exchange interactions occur. I briefly review the analogy between the singlet and triplet types of pairing, as well as draw some general conclusions about the pairing induced by these exchange interactions. The general discussion is also illustrated with a concrete case of a two-dimensional lattice with the spin triplet pairing.
\end{abstract}

Key words: $t-J$ model, real space pairing, strong correlations, singlet pairing, triplet pairing, superconductivity

PACS: $71.10 . F d, 74.20 . M n, 71.27 .+a, 74.25 . D w, 74.72 .-h$

\section{Introduction}

The $\mathrm{t}-\mathrm{J}$ model contains a very attractive idea of spin-singlet pairing induced by electron correlations and taking place in high-temperature superconducting cuprates [1,2], although some questions remain whether the actual pairing should also include explicitly the hybrid $2 p-3 d$ pairing induced by interactions of the Kondo-type [3,4]. What is fundamental in this approach is the crucial role of a strong Coulomb repulsion between electrons in these narrow-band systems, which leads to the antiferromagnetic intersite interaction, yielding in turn the effective pairing in real space. Even the effect of the atomic disorder leading to an enhancement of the pairing on local scale can be accounted for semiquantitatively [5]. The question of the pseudogap appearance in the underdoped systems is still under debate, but it may be related to the residual antiferromagnetic correlations in a strongly disordered medium, induced by both the doping and the correlations of charge and spin type (stripes). In general, the nature of the normal state is understandable to a lesser extent than the condensed superconducting state in the two spatial dimensions. The role of the third dimension (coupling between the planes), as well as that of electron-lattice coupling, still remains at a further distance.

On the other hand, the first unambiguous defection of spin-triplet superconductivity in the ferromagnetic phase [6,7] did not spark a corresponding modelling of the spin-triplet pairing based on an analogous approach, as it deserved (see, however $[8,9]$ ). The systems such as $\mathrm{UGe}_{2}$ or $\mathrm{URhGe}$ may not be as strongly correlated as the cuprates or heavy fermions, but still it is important to explore the generalized $\mathrm{t}-\mathrm{J}$ model for orbitally degenerate systems. This topic will be briefly overviewed here. In this connection, one should mention the just discovered Fe-pnictide superconductors, which are orbitally degenerate systems, so they may be good candidates for a singlet-triplet superconducting transition as a function of composition (i.e. concentration of $3 d$ electrons). The presence of such a transition would provide an unambiguous evidence for the role of kinetic exchange interactions in real-space pairing in orbitally degenerate narrow-band systems. The main purpose of this paper is to comment of two nonstandard features of the $\mathrm{t}-\mathrm{J}$ model and then, to turn attention to an effective $\mathrm{t}-\mathrm{J}$ model for orbitally degenerate systems.

*E-mail: ufspalek@if.uj.edu.pl; URL: http://th-www.if.uj.edu.pl/ztms/jspalek_een.htm 


\section{2. $\mathrm{t}-\mathrm{J}$ model}

The $\mathrm{t}-\mathrm{J}$ model with inclusion of antiferromagnetic kinetic exchange and the three-site hopping was derived over 3 decades ago [10]. Starting from the Hubbard model, it has the following form for an electron moving in the lower Hubbard band

$$
\begin{aligned}
& P_{0} \tilde{H} P_{0}=\sum_{i j \sigma} t_{i j} a_{i \sigma}^{\dagger}\left(1-n_{i \bar{\sigma}}\right) a_{j \sigma}\left(1-n_{j \bar{\sigma}}\right)+\sum_{i j}\left(2 t_{i j}^{2} / U\right)\left[\mathbf{S}_{i} \cdot \mathbf{S}_{j}-\frac{1}{4} \sum_{\sigma \sigma^{\prime}} n_{i \sigma}\left(1-n_{i \bar{\sigma}}\right) n_{j \sigma^{\prime}}\left(1-n_{j \bar{\sigma}^{\prime}}\right)\right] \\
& \quad+\sum_{i j k \sigma} \frac{t_{i j} t_{j k}}{U}\left[a_{i \sigma}^{\dagger}\left(1-n_{i \bar{\sigma}}\right) n_{j \bar{\sigma}}\left(1-n_{j \sigma}\right) a_{k \sigma}\left(1-n_{k \bar{\sigma}}\right)-a_{i \sigma}^{\dagger}\left(1-n_{i \bar{\sigma}}\right) \mathbf{S}_{j}^{\bar{\sigma}} a_{k \bar{\sigma}}\left(1-n_{k \sigma}\right)\right]
\end{aligned}
$$

The first term represents the hopping between the neighbors $i$ and $j$ ( $t_{i j} \neq 0$ for $i \neq j$ only), the second one represents the so-called kinetic exchange interaction with the fermionic representation of the spins: $\mathbf{S}_{i} \equiv\left(S_{i}^{+}, S_{i}^{-}, S_{i}^{z}\right) \equiv\left(S_{i}^{\sigma}, S_{i}^{z}\right) \equiv\left(a_{i \uparrow}^{\dagger} a_{i \downarrow}, a_{i \downarrow}^{\dagger} a_{i \uparrow},\left(n_{i \uparrow}-n_{i \downarrow}\right) / 2\right)$, and $\bar{\sigma} \equiv-\sigma$. The three processes composing the effective Hamiltonian are depicted schematically in figure 1.

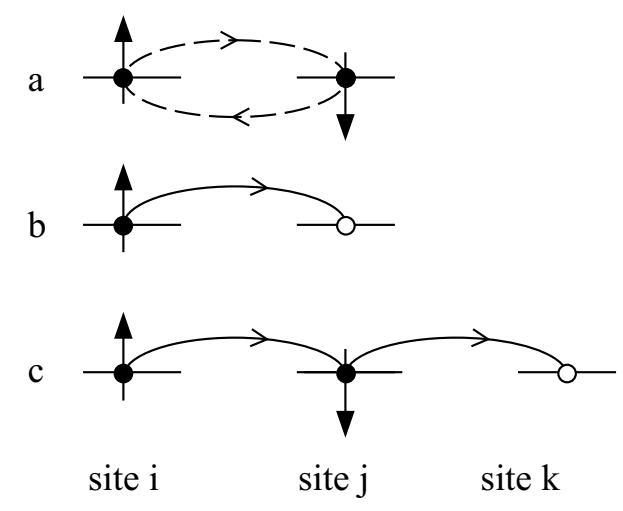

Figure 1. Possible hopping processes in the lower Hubbard subband: a) virtual hopping between singly occupied sites that leads to an antiferromagnetic (kinetic) exchange interaction between the neighboring sites; b) two-site hopping between empty and singly occupied sites (singleparticle hopping); and c) between the singly occupied and empty sites via a singly occupied site.

The whole dynamics can be expressed in a closed form by introducing projected fermionic operators:

$$
\begin{gathered}
b_{i \sigma}^{\dagger} \equiv a_{i \sigma}^{\dagger}\left(1-n_{i \bar{\sigma}}\right), b_{i \sigma} \equiv a_{i \sigma}\left(1-n_{i \bar{\sigma}}\right), \\
\nu_{i \sigma} \equiv b_{i \sigma}^{\dagger} b_{i \sigma}=n_{i \sigma}\left(1-n_{i \bar{\sigma}}\right), \nu_{i}=\sum_{\sigma} \nu_{i \sigma},
\end{gathered}
$$

and then the Hamiltonian assumes the following closed form:

$$
\begin{aligned}
P_{0} \tilde{H} P_{0} & =\sum_{i j \sigma} t_{i j} b_{i \sigma}^{\dagger} b_{j \sigma}+\sum_{i j}\left(\frac{2 t_{i j}^{2}}{U}\right)\left(\mathbf{S}_{i} \cdot \mathbf{S}_{j}-\frac{1}{4} \nu_{i} \nu_{j}\right) \\
& +\sum_{i j k \sigma} \frac{t_{i j} t_{j k}}{U}\left(b_{i \sigma}^{\dagger} \nu_{j \bar{\sigma}} b_{k \sigma}-b_{i \sigma}^{\dagger} S_{j}^{-\sigma} b_{k \bar{\sigma}}\right) .
\end{aligned}
$$

As the projected operators $\left\{b_{i \sigma}^{\dagger}\right\}$ and $\left\{b_{i \sigma}\right\}$ obey the non-fermion anticommutation relations from one side and the hopping term does not commute with the second order $\left(\sim t^{2} / U\right)$ part, the dynamics of this model is highly nontrivial. Physically, this is because the kinetic-energy part vanishes in the limit of the Mott insulator $\left(\left\langle\nu_{i}\right\rangle=1\right)$, but in the metallic phase the first two terms may be of the first two terms can be of comparable magnitude. 
An important comment can be made at this point. Namely, in the low-dimensional systems, the Coulomb interaction screening is less effective (particularly for a small number of holes in the Mott insulator). Therefore, the intersite interaction part

$$
\frac{1}{2} \sum_{i \neq j} K_{i j} n_{i} n_{j}
$$

can be regarded as important, particularly near the Mott-insulator limit. In deriving the effective Hamiltonian, the coupling constant for the second-order terms is then $\sim t_{i j} t_{j k} /\left(U-K_{i j}\right)$, which means that the effective spin-spin interaction becomes stronger. This is not so good for the real space pairing, since one has to add to the effective Hamiltonian (3) an intersite repulsion $(1 / 2) \sum K_{i j} \nu_{i} \nu_{j}$ and if $K_{i j}>4 t_{i j}^{2} /\left(U-K_{i j}\right)$, then the spin-spin coupling is not sufficient to produce the attractive interaction, i.e., to produce bound pairs in the ordinary sense. To see this more explicitly, we introduce the intersite spin-singlet pairing operators as follows:

$$
B_{i j}^{\dagger} \equiv \frac{1}{\sqrt{2}}\left(b_{i \uparrow}^{\dagger} b_{j \downarrow}^{\dagger}-b_{i \downarrow}^{\dagger} b_{j \uparrow}^{\dagger}\right), \quad B_{i j}=\left(B_{i j}^{\dagger}\right)^{\dagger}=-\frac{1}{\sqrt{2}}\left(b_{i \uparrow} b_{j \downarrow}-b_{i \downarrow} b_{j \uparrow}\right),
$$

and hence (3) takes the most compact form

$$
P_{0} \tilde{H} P_{0}=\sum_{i j \sigma} t_{i j} b_{i \sigma}^{\dagger} b_{j \sigma}-\sum_{i j k} \frac{2 t_{i j} t_{j k}}{U} B_{i j}^{\dagger} B_{k j}+\frac{1}{2} \sum_{i \neq j} K_{i j} \nu_{i} \nu_{j}
$$

The destructive role of the intersite Coulomb repulsion is usually ignored (see also below). However, taken literally, it simply can preclude any bound-state formation associated with the realspace pairing, i.e. when $\left\langle B_{i j}\right\rangle \neq 0$. To overcome this intersite repulsion, a local lattice-distortion induced attraction can play an important role. An elementary argument for the effect of the distortion on the effective attractive interaction is briefly analyzed below $[10,11]$.

\section{Bond distortion and the effective attractive interaction in $\mathrm{t}-\mathrm{J}$ model}

In the narrow-band limit, the hopping integral diminishes roughly exponentially with the increasing inter-atomic distance $\mathbf{R}_{i j}$. On the contrary, in this asymptotic limit, the intersite long-range Coulomb repulsion can be approximated to the first order by its classical expression $K_{i j} \approx \mathrm{e}^{2} / R_{i j}$. Therefore, the change of the system energy $\delta H$ under the effect of the classical bond distortion $\delta \mathbf{R}_{i j}$ is mainly obtained due to the change of the intersite Coulomb energy via $\left(\delta K_{i j} / \delta \mathbf{R}_{i j}\right) \cdot \delta \mathbf{R}_{i j}$, i.e. as

$$
\frac{\delta K_{i j}}{\delta \mathbf{R}_{i j}} \simeq-\frac{\mathrm{e}^{2}}{2 R_{i j}^{2}} \frac{\mathbf{R}_{i j}}{R_{i j}}
$$

We can write down the total system energy with the inclusion of the lattice distortion in the form (we omit three-site terms for simplicity):

$$
\begin{aligned}
\tilde{H}= & P_{0} \tilde{H} P_{0}+\delta H=\sum_{i j \sigma} t_{i j} b_{i \sigma}^{\dagger} b_{j \sigma}+\sum_{i j} \frac{2 t_{i j}^{2}}{U-K_{i j}} B_{i j}^{\dagger} B_{i j}+\frac{1}{2} \sum_{i \neq j} K_{i j} \nu_{i} \nu_{j} \\
& -\frac{1}{2} \sum_{i \neq j} W_{i j} \frac{\mathbf{R}_{i j} \cdot \delta \mathbf{R}_{i j}}{R_{i j}^{2}} \nu_{i} \nu_{j}+\frac{1}{2} \sum_{i \neq j} \gamma_{1}\left(\delta \mathbf{R}_{i j}\right)^{2}+\gamma_{2} \sum_{\alpha \neq \beta} \delta R_{i j}^{\alpha} \delta R_{i j}^{\beta},
\end{aligned}
$$

where $W_{i j} \simeq K_{i j} \simeq \mathrm{e}^{2} /\left(2 R_{i j}\right), \gamma_{1}$ and $\gamma_{2}$ are the diagonal and off-diagonal elastic constants, respectively. Minimizing the energy with respect to classical bond distortions $\delta \mathbf{R}_{i j}$, one obtains [11] the following effective Hamiltonian including both strong correlations and the attractive interaction induced by the particle motion (e.g. holes in the Mott insulator). Assuming that we have an 
isotropic distortion accompanying the hole motion in the planar case, we obtain the following generalized t- $\mathbf{J}$ Hamiltonian (up to an irrelevant term):

$$
\tilde{H}=\sum_{i j \sigma} t_{i j} b_{i \sigma}^{\dagger} b_{j \sigma}-\sum_{i j}\left(\frac{4 t_{i j}^{2}}{U-K_{i j}}+\frac{1}{4} \frac{W_{i j}^{2}}{R_{i j}^{2} \gamma_{1}}-K_{i j}\right) b_{i \uparrow}^{\dagger} b_{j \downarrow}^{\dagger} b_{j \downarrow} b_{i \uparrow},
$$

or equivalently,

$$
\tilde{H}=\sum_{i j \sigma}\left(t_{i j} b_{i \sigma}^{\dagger} b_{j \sigma}-\frac{1}{2} J_{i j} \nu_{i \sigma} \nu_{j \bar{\sigma}}\right),
$$

where $J_{i j}$ expresses the sum in the bracket in (8). This is the spin-dependent version of an attractive density-density interaction [12], which still expresses the complementary character of antiferromagnetic spin-spin correlations and the intersite singlet pairing (note that $\nu_{i \sigma} \nu_{j \bar{\sigma}}=b_{i \sigma}^{\dagger} b_{j \bar{\sigma}}^{\dagger} b_{j \bar{\sigma}} b_{i \sigma}$ ).

The essence of the above argument is as follows. The inclusion of a bond distortion associated with the particle density-density correlation $\left(\left\langle\nu_{i} \nu_{j}\right\rangle \neq 0\right)$ results in an effective $\mathrm{t}-\mathrm{J}$ model of the type (9). The bond-distortion contribution can be large as the ratio of the bare intersite Coulomb interaction $\left(W_{i j}\right)$ to $\gamma_{1} R_{i j}^{2}$ can be large and therefore, together with the kinetic-exchange contribution $\left(\sim t_{i j}^{2}\right)$, it can overcome the intersite repulsion $\sim K_{i j}$ between the particles with the opposite spins. In this manner, the $\mathrm{t}-\mathrm{J}$ model looks like a model of real-space pairing in a single narrow band, in which the Cooper pairs are formed as bound states in an attractive potential. In the absence of the bond distortion, the Cooper-pair and the condensed states are formed purely by the electronic correlations induced by the repulsive Coulomb interactions.

\section{Hybrid singlet $d-p$ vs. $d-d$ pairing: A brief comment}

The magnetism of strongly correlated electrons started with the pioneering introduction of Hubbard model by Anderson [13,14] in the context of antiferromagnetic ordering of transitionmetal oxides. In this treatment, the role of the filled $2 p$ states due to oxygen $\left(\mathrm{O}^{2-}\right)$ is passive, i.e., to mediate the effect of $d-d$ interaction between the magnetic ions. The role of oxygen is not only passive in metal-insulator transition, for example [15]. The question is what happens if the state is metallic and the $p$ bands become partially filled and the $p-d$ hybridization starts playing an active role in the dynamics of the metallic phase. The passive role of the $p$ electrons has been assumed by Zhang and Rice [16] in their intuitive justification of the $\mathrm{t}-\mathrm{J}$ model by assuming that the Kondolike (Zhang-Rice) singlet is so tightly bound that the $p-d$ antiferromagnetic correlations lead to effective $d-d$ interactions. The situation seems very likely for the cuprates (see however e.g. [17]). However, it is certainly not the case for the heavy fermions, where the hybridization between the strongly correlated $4 f$ states (of e.g. $\mathrm{Ce}^{3+}$ ion) and the $5 d-6 s$ valence states destabilizes the $4 f$ localized moments, and in effect leads to the heavy-fermion behavior, and can at the same time become the source of hybrid pairing $[8,10]$. Parenthetically, the situation may be different in heavyfermion uranium compounds such as $\mathrm{UPt}_{3}$, where the $\mathrm{U}^{4+}$ (approximately $5 f^{2}$ ) configuration may lead, in conjunction with the Hund's rule coupling among $5 f$ electrons, to the triplet pairing. This type of situation will be considered in the next section.

\section{Real-space pairing in the doubly degenerate band}

\subsection{The physical situation and the model}

As we have seen in the two foregoing sections, the real space pairing in the orbitally nondegenerate case leads, even in the two-band situation, to the singlet correlations and to the corresponding bound Cooper pairs [19]. Now we discuss the situation in the orbitally degenerate Hubbard model and include the Hund's rule coupling directly, as well as highlight its role in the strong-correlation limit, where both ferro- and antiferromagnetic kinetic exchange interactions appear at a proper band filling. The purpose of this discussion is to show that the real-space spin-triplet pairing is theoretically as feasible as is the singlet pairing in the single-band case. 
The starting Hamiltonian for such a two-band case has the following form

$$
\begin{aligned}
H= & \sum_{i j l l^{\prime} \sigma} t_{i j}^{l l^{\prime}} a_{i l \sigma}^{\dagger} a_{j l^{\prime} \sigma}+U \sum_{i l} n_{i l \uparrow} n_{i l \downarrow} \\
& +\sum_{i l \neq l^{\prime}}\left[\left(U^{\prime}-\frac{1}{2} J_{\mathrm{H}}\right) n_{i l} n_{i l^{\prime}}-J_{\mathrm{H}} \mathbf{S}_{i l} \cdot \mathbf{S}_{i l^{\prime}}+J_{\mathrm{H}} a_{i l \uparrow}^{\dagger} a_{i l \downarrow}^{\dagger} a_{i l^{\prime} \downarrow} a_{i l^{\prime} \uparrow}\right],
\end{aligned}
$$

where $l, l^{\prime}=1,2$ are the orbital indices, the first and the second terms are respectively the hopping and the Hubbard parts, whereas the last three terms describe the interorbital Coulomb repulsion, the ferromagnetic Hund's rule and the pair-electron-hopping terms, respectively (both have the same amplitude $J_{\mathrm{H}}$ ). Without a loss of generality, one may assume that $U^{\prime}=U-2 J_{\mathrm{H}}$ which holds at least for $3 d e_{g}$ orbitals. To simplify the discussion, we assume that the hopping is diagonal and the same, i.e. $t_{i j}^{l l^{\prime}}=t_{i j} \delta_{l l^{\prime}}$ to avoid the complications associated with the interorbital hopping part (with $l \neq l^{\prime}$ ) and the corresponding exchange.

The model in the strong-correlation limit $U \gg J_{\mathrm{H}} \gg\left|t_{i j}\right|$ leads to ferromagnetic Mott insulator (with possible orbital ordering) for quarter-filled band $(n=1)$ and to the ordinary antiferromagnetic Mott insulator at half filling $(n=2)$ [20-22]. The reason for ferromagnetism near $n \sim 1$ is due to the fact that the dominant virtual hopping process between two neighboring sites takes place to the local spin-triplet state in the intermediate state. This intermediate on-site spin-triplet state does not appear in an orbitally nondegenerate model. On the other hand, for the case of two electrons per atom $(n=2)$ only the virtual-hopping processes leading to the spin-singlet pair configuration in the intermediate state are allowed, so the kinetic exchange interaction is antiferromagnetic, in a direct analogy to that in a single-band case. This gradual transition from paramagnetism through ferromagnetism to antiferromagnetism has been observed in a series of isostructural compounds $\mathrm{FeS}_{2}-\mathrm{CoS}_{2}-\mathrm{NiS}_{2}$ and their mixtures, as then a doubly degenerate band of $e_{g}$ symmetry is filled when we go from $\mathrm{FeS}_{2}(n=0)$, through $\mathrm{CoS}_{2}(n=1)$, to $\mathrm{NiS}_{2}(n=2)$. At an intermediate composition $1<n<2$ a spin-glass-like state appears and is caused by the competing ferro- and antiferromagnetic interactions [23].

\subsection{Spin-triplet paired states}

The model of the real-space pairing is built in a direct correspondence to the theory of kinetic exchange. We consider the case of identical orbitals, for which the nearest hopping integrals are the same $t \equiv t_{\langle i j\rangle}$. The relevant virtual hopping processes are depicted in figure 2 . The effective $\mathrm{t}-\mathrm{J}-\mathrm{J}_{\mathrm{H}}$ model takes the form for $n \leqslant 1[24]$

$$
\begin{aligned}
P_{0} \tilde{H} P_{0}= & \sum_{i j l \sigma} t_{i j} b_{i l \sigma}^{\dagger} b_{j l \sigma}-\frac{4}{U-3 J} \sum_{\substack{i \neq j \neq r \\
m=-1,0,1}} t_{i j} t_{j r} B_{i j m}^{\dagger} B_{i j n} \\
& -\frac{4}{U-J} \sum_{\substack{i \neq j \neq r \\
\text { ij }}} t_{i j} t_{j r} C_{i j 0}^{\dagger} C_{r j 0}-\frac{2}{U} \sum_{\substack{i \neq j \neq r \\
m= \pm 1}} t_{i j} t_{j r} C_{i j m}^{\dagger} C_{j r m} \\
& +\frac{4 J}{U^{2}} \sum_{\substack{i \neq j \neq r \\
m=-m= \pm 1}} t_{i j} t_{j r} C_{i j m}^{\dagger} C_{r j \bar{m}},
\end{aligned}
$$

where $b_{i 1 \sigma}^{\dagger} \equiv a_{i 1 \sigma}^{\dagger}\left(1-n_{i 1 \bar{\sigma}}\right)\left(1-n_{i 2 \bar{\sigma}}\right)$, etc. The pairing operators with $-1,0,+1$ are the projected interorbital pairing operators for the spin-triplet states with $S^{z}=1,-1$, and 0 , respectively, i.e.

$$
\begin{aligned}
& B_{i j 1}^{\dagger} \equiv b_{i 1 \uparrow}^{\dagger} b_{j 2 \uparrow}^{\dagger} \\
& B_{i j-1}^{\dagger} \equiv b_{i 1 \downarrow}^{\dagger} b_{j 2 \downarrow}^{\dagger} \\
& B_{i j 0}^{\dagger} \equiv \frac{1}{\sqrt{2}}\left(b_{i 1 \uparrow}^{\dagger} b_{j 2 \downarrow}^{\dagger}-b_{i 1 \downarrow}^{\dagger} b_{j 2 \uparrow}^{\dagger}\right) \quad \text { for } \quad m=S^{z}=0 \text {. }
\end{aligned}
$$




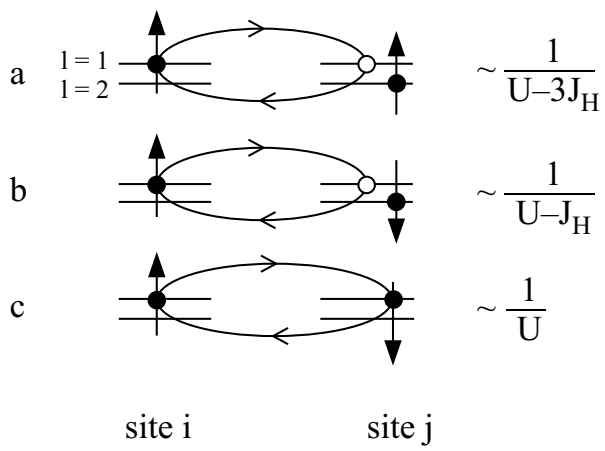

Figure 2. Possible virtual hopping processes leading to the three pairing terms shown in the text (the corresponding denominators in equation (11) are written on the right).

The operators $C$ are the spin-singlet pairing operators which we call the orbital pairing operators. That is why they are defined as follows:

$$
\begin{array}{rlrl}
C_{i j 1}^{\dagger} & =\frac{1}{\sqrt{2}}\left(b_{i 1 \uparrow}^{\dagger} b_{j 1 \downarrow}^{\dagger}-b_{i 1 \downarrow}^{\dagger} b_{j 1 \uparrow}^{\dagger}\right) & m & \equiv L^{z}=1, \\
C_{i j \overline{1}}^{\dagger}=\frac{1}{\sqrt{2}}\left(b_{i 2 \uparrow}^{\dagger} b_{j 2 \downarrow}^{\dagger}-b_{i 2 \downarrow}^{\dagger} b_{j 2 \uparrow}^{\dagger}\right) & m & \equiv L^{z}=-1 \\
C_{i j 0}^{\dagger} & \equiv \frac{1}{2}\left(b_{i 1 \uparrow}^{\dagger} b_{j 2 \downarrow}^{\dagger}+b_{i 2 \uparrow}^{\dagger} b_{j 1 \downarrow}^{\dagger}-b_{i 1 \downarrow}^{\dagger} b_{j 2 \uparrow}^{\dagger}-b_{i 2 \downarrow}^{\dagger} b_{j 1 \uparrow}^{\dagger}\right) & m & \equiv L^{z}=0 .
\end{array}
$$

The pairing operators $C_{i j m}^{\dagger}$ are both intraorbital $(m= \pm 1)$ and interorbital $(m=0)$ character. We see that the spin-triplet term is dominant for $n \leqslant 1$. One should also note that the intersite projected pairing operators (12) replace their intraatomic correspondant [25,26], which appear in the limit of low and intermediate correlations and are related directly to the intraatomic ferromagnetic Hund's rule coupling. Therefore, our work shows that the Hund's rule coupling can lead not only to ferromagnetism (e.g. when the Stoner criterion is fulfilled), but also to spin-triplet superconductivity in both moderate- and strong-correlation regimes. It is tempting to propose that the spin-triplet superconductivity may appear in pure samples of e.g. $\mathrm{CoS}_{2}$ under pressure, when ferromagnetism is suppressed. The question of a coexistence of the spin-triplet superconductivity and ferromagnetism is a separate issue.

\section{Phase diagram of spin-triplet paired states}

Finally, we provide an overall phase diagram of the spin-triplet phases and leave the details to a separate paper [27]. We consider a two-dimensional square lattice case with the isotropic gap $\left(\Delta_{m}=\Delta\right)$. The superconducting order parameter is chosen in the form

$$
\Delta_{m \mathbf{k}}=\Delta_{f}\left(\cos k_{x}+\mathrm{e}^{\mathrm{i} \alpha} \cos k_{y}\right) .
$$

In the slave-boson language, originally developed for t-J model [28], this fermionic gap amplitude $\Delta_{f}$ is multiplied by the slave boson occupancy $\Delta_{b}=\left\langle b_{i}^{\dagger} b_{j}\right\rangle \approx b^{2}$, and the superconducting state is achieved when both the fermionic parameter $\Delta_{f} \sim\left\langle B_{i j m}^{\dagger}\right\rangle$ and the bosonic amplitude $\Delta_{b}$ are simultaneously different from zero. The solutions then are of extended $s$-wave type (if $\alpha=0$ ), $s+\mathrm{i} d$ type (when $\alpha=\pi / 2$ ), and of $d$ type (if $\alpha=\pi$ ). In general, when $\alpha \neq(0, \pi / 2, \pi)$ the state is called $s-d$ mixed state. The phase diagram representing the stable phases in the ground state is shown in figure 3 on the plane $\delta \equiv 1-n$ versus $J_{\mathrm{H}} / U$. The other parameter is $|t| / U=0.2$. For $\delta=0$, the system is a ferromagnetic insulator, so the paired states evolve from a ferromagnetic Mott insulator. Note the presence of a usual "dome" of $d$-wave superconductivity, located in the interval $0.04 \leqslant \delta \leqslant 0.13$. In the regime of low electron concentration $\delta \geqslant 0.20$, an ordinary gapless ( $s$-wave) type of pairing takes place. It should be underlined that in order to determine stability of 
the phases we have to solve the system of equations for $\Delta_{f}, \Delta_{b}$, and the chemical potential $\mu$, for a given phase factor $\alpha$. The details of this cumbersome analysis will be discussed elsewhere [27].

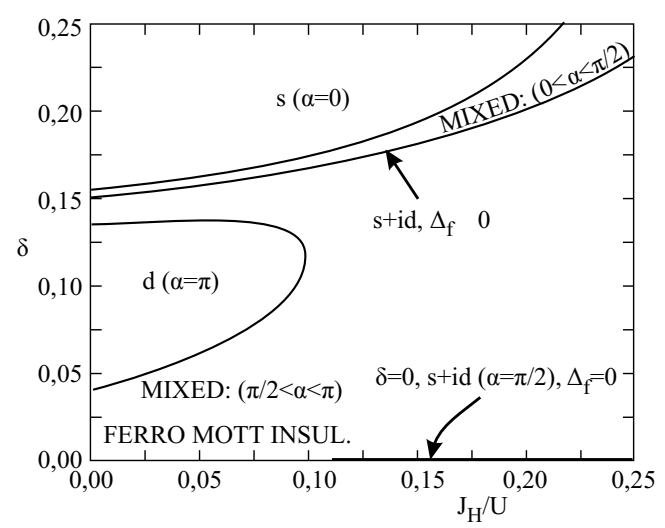

Figure 3. Paired ground states on the plane number of holes (per site) in ferromagnetic Mott insulator versus the relative strength of Hund's rule coupling. For details see the main text.

\section{Outlook}

In this brief overview we have first discussed the effect of the classical bond distortion in deriving an effective form of the spin-singlet pairing for strongly correlated electrons in a nondegenerate band. The inclusion of the lattice allows to draw a distinction between the pairing achieved by the effective attractive interaction and the pairing achieved solely by the correlations induced by the purely repulsive Coulomb interaction. The role of hybrid $2 p-3 d$ or $5 d-4 f$ correlations in deriving respectively the effective spin-singlet pairing for high temperature and heavy-fermion systems is noted in passing. The main message of our paper is though to indicate that an analogous type of pairing can take place for electrons in orbitally degenerate and strongly correlated systems. Namely, we have discussed the hole pairing in the doped ferromagnetic Mott insulator, with the doping $\delta=1-n>0$. It would be interesting to see if such a situation is possible in one of the newly synthetized materials. This new possibility at least extends the theoretical applicability of the $\mathrm{t}-\mathrm{J}$ model.

\section{Acknowledgements}

This work is dedicated to Professor Ihor Stasyuk on the occasion of his 70th Bithday. His papers on the Hubbard atomic representation belong to the pioneering works on strongly correlated systems. I also acknowledge the Grant from the Ministry of Science and Higher Education of Poland, No. 1 P03B 029 001. The project is being carried out under the auspices of the European COST P-16 Projects Emergent Behaviour of Correlated Matter. The author is also grateful to Drs. Andrzej Klejnberg and Robert Podsiadły for discussions and technical help. This project is also partially supported by the Marie Curie TOK Grant MTDK-CT-2004-517186 Correlations in Complex Systems (COCOS).

\section{References}

1. Ruckenstein A.F., Hirschfeld P., Appel J., Phys. Rev. B, 1987, 36, 857; Plakida N.M., Yushankhai V.Yu., Stasyuk I.V., Physica C, 1989, 160, 80.

2. Anderson P.W. Frontiers and Borderlines in many-Particle Physics, Eds. Broglia R.A., Schrieffer J.R. North-Holland, Amsterdam, 1988, p. 1ff.

3. Spałek J., Phys. Rev. B, 1988, 38, 208.

4. Spałek J., Gopalan P., J. Phys. (France), 1989, 50, 2869. 
5. Maśka M. et al., Phys. Rev. Lett., 2007, 99, 147006.

6. Saxena S.S. et al., Nature, 2000, 406, 587.

7. Aoki D. et al., Nature, 2001, 413, 613.

8. Klejnberg A., Spałek J., Phys. Rev. B, 2000, 61, 15542.

9. Spałek J., Wróbel P., Wójcik W. Ruthenate and Rutheno-Cuprate Superconductors, Eds. C. Noce et al. Springer Verlag, Berlin, 2002, vol. 603, pp. 60-75.

10. For recent review see e.g.: Spałek J., Acta Phys. Polon. A, 2007, 111, 409.

11. Spałek J., to be submitted; Spałek J., Oleś A.M., Physica B, 1977, 86-88, 375; Chao K.A., Spałek J., Oleś A.M., J. Phys. C, 1977, 10, L271.

12. Micnas R., Ranninger J., Robaszkiewicz S., Rev. Mod. Phys., 1990, 62, 113.

13. Anderson P.W., Phys. Rev. B, 1959, 115, 2.

14. Anderson P.W. Solid State Physics, Eds. Seitz F. and Turbull D. Academic Press, New York, 1963, vol. 14, p. $99 \mathrm{ff}$.

15. Zaanen J., Sawatzky G.A., Allen J.W., Phys. Rev. Lett., 1985, 55, 418; J. Magn. Magn. Mat., 1986, 55-57, 607.

16. Zhang F.C., Rice T.M., Phys. Rev. B, 1988, 37, 3759.

17. Eremin M., Rigamonti A., Phys. Rev. Lett., 2002, 88, 037002.

18. Spałek J., Maśka M., Mierzejewski M., Kaczmarczyk J., submitted for publication.

19. Byczuk K., Spałek J., Wójcik W., Phys. Rev. B, 1992, 46, 14134.

20. Kugel K.I., Khomskii D.I., Sov. Phys. - JETP, 1973, 37, 725.

21. Cyrot M., Lyon-Caen C., J. Phys. (France), 1975, 36, 253.

22. Spałek J., Chao K.A., J. Phys. C: Sol. State Phys., 1980, 13, 5241.

23. Ogawa S., J. Appl. Phys., 1979, 50, 2308.

24. Klejnberg A., Ph. D. Thesis, Jagiellonian University, Kraków 2006, unpublished.

25. Klejnberg A., Spałek J., J. Phys.: Condens. Matter, 1999, 11, 6553.

26. Spałek J., Phys. Rev. B, 2001, 63, 104513.

27. Klejnberg A., Spałek J., in preparation.

28. Suzumura Y., Hasegawa Y., Fukuyama H., J. Phys. Soc. Jpn., 1988, 57, 401, 2768; Fukuyama H., Hasegawa Y., Suzumura Y., Physica C, 1988, 153-155, 1630.

\title{
Ефективна $\mathrm{t}-\mathrm{J}$ модель спарювання: синглет проти триплету
}

\author{
Й.Спалек \\ Інститут фізики ім. Мар'яна Смолуховського, Ягелонський університет, вул. Реймонта 4, Краків \\ 30-059, Польща
}

Отримано 30 травня 2008 р., в остаточному вигляді - 18 червня 2008 р.

\begin{abstract}
Часто $\mathrm{t}-\mathrm{J}$ модель розглядається як канонічна модель спінового синглетного спарювання, що створюється кінематичною обмінною взаємодією, яка також відповідає за антиферомагнітне впорядкування у сильно скорельованих вузькозонних системах. У орбітально вироджених системах одночасно присутні феромагнітна та антиферомагнітна кінематичні обмінні взаємодії. Робиться короткий огляд аналогії між синглетним та триплетним типами спарювання, а також окреслюються деякі загальні висновки щодо спарювання, яке створюється цими обмінними взаємодіями. Загальний розгляд також доповнюється обговоренням випадку двовимірної гратки зі спіновим триплетним спарюванням.
\end{abstract}

Ключові слова: $t-J$ модель, спарювання у дійсному просторі, сильні кореляції, синглетне спарювання, триплетне спарювання, надпровідність

PACS: 71.10.Fd, 74.20.Mn, 71.27.+a, 74.25.Dw, 74.72.- $h$ 